\title{
Análisis del uso de asistentes virtuales en el aula como recurso complementario en la práctica docente
}

\author{
María del Sol Pérez ${ }^{1}$, Claudia Villalonga² ${ }^{2}$ Alberto Guillén ${ }^{1}$, Oresti Baños ${ }^{1}$ \\ 1 Departamento de Arquitectura y Tecnología de Computadores, Universidad de Granada \\ Granada, España \\ \{aguillen, oresti\}@ugr.es \\ 2 Escuela Superior de Ingeniería y Tecnología, Universidad Internacional de La Rioja \\ Logroño, España \\ Claudia.villalonga@unir.net
}

\begin{abstract}
Resumen. Actualmente el profesorado dispone de múltiples recursos para impartir su docencia e intentar hacer sus clases más atractivas. No obstante, el simple hecho de introducir las TIC en la docencia no es condición suficiente para la mejora de la práctica docente, por lo que es necesario evaluar su impacto y beneficios. El principal objetivo del presente trabajo es investigar la eficacia de las respuestas generadas por los asistentes virtuales con voz, como Google Nest o Amazon Echo, utilizados como material de apoyo para el docente en la asignatura de Tecnología en la Educación Secundaria Obligatoria. La investigación se ha llevado a cabo con alumnado real. Se ha comprobado que el uso de los asistentes virtuales con voz para la resolución de dudas dentro de la materia de Tecnología es limitado, ya que dichos asistentes no han sido capaces de responder todas las consultas planteadas por el alumnado. Además se constata que la validez y precisión en las respuestas proporcionadas también son limitadas. La percepción del alumnado en cuanto a la utilidad de estos asistentes para resolver sus dudas es bastante homogénea, y si bien se considera positiva, en múltiples casos las respuestas recibidas no han resultado suficientemente aclaratorias.
\end{abstract}

Palabras Clave: Asistente virtual, Tecnología, Google Nest, Amazon Echo.

\begin{abstract}
Currently, teachers have multiple resources to teach and try to make their classes more attractive. However, introducing ICT in teaching is not enough for the improvement of teaching practice, thus making it necessary to evaluate its impact and benefits. The main objective of this work is to investigate on the effectiveness of responses generated by virtual assistants with voice (smart speakers) as Google Nest or Amazon Echo, which are used as support material for teachers in the Technology course in High School. This research has found that the use of smart speakers for answering questions within the scope of the Technology course is limited, since these assistants have not been able to answer all the questions raised by students. In addition, it is noted that the validity and accuracy of the answers provided is also limited. The students' perception of the usefulness of these assistants to solve their doubts is quite homogeneous, and although it is considered positive, in many cases the answers received have not been sufficiently clarifying.
\end{abstract}

Keywords: Virtual Assistant, Technology, Google Nest, Amazon Echo. 


\section{Introducción}

En la actualidad, las Tecnologías de la Información y la Comunicación (TIC) se encuentran presentes en múltiples entornos de la vida diaria de una persona, pudiendo encontrarse en el trabajo, en el hogar, en el colegio e incluso en el propio vehículo. La fuerte presencia de las TIC en nuestras vidas, unido a su desarrollo y su evolución, ha originado la adaptación progresiva del ser humano a un mundo totalmente digitalizado. Por este motivo, los centros educativos se encuentran ante un gran desafío, ya que resulta necesario introducir la utilización de las TIC dentro del sistema de enseñanza. A pesar de la dificultad que supone este reto, la introducción de las TIC en el aula, en casa y como parte del diseño curricular tiene una gran relevancia dentro del ámbito educativo.

La integración de las TIC en la actividad docente desarrollada hoy en día, supone un derecho para el alumnado ya que se ha convertido en una demanda social, una necesidad para el profesorado y una obligación para la administración educativa. Cabe destacar que la utilización de las TIC en la enseñanza, favorece y ayuda al alumnado en su aprendizaje, aumenta su interés y creatividad, contribuye al crecimiento de su motivación, potencia el trabajo en equipo, mejora la capacidad de solventar problemas, fortalece su autoestima y permite un mayor aumento en la autonomía en cuanto al aprendizaje se refiere [1].

Una rama de las TIC que está en pleno apogeo es la inteligencia artificial. Para que una máquina adquiera habilidades humanas lo primero que hay que otorgarle es una voz, además de la capacidad de poder hablar. Es aquí donde investigadores y empresas como Amazon, Google o Microsoft tienen un papel muy importante, ya que después de muchos esfuerzos y arduo trabajo han conseguido que los humanos puedan interactuar con una máquina como si de otro humano se tratase. Estas máquinas son conocidas como asistentes virtuales con voz y están al alcance de cualquiera ya que su coste no es muy elevado. Los asistentes virtuales con voz tienen múltiples funcionalidades que se activan a través de órdenes con la voz, de forma que el usuario del asistente solo tiene que pedir lo que necesite mediante el habla. Entre las diversas funcionalidades de las que dispone es conveniente destacar la de programar citas en el calendario o fijar alarmas, reproducir música, realizar compras, controlar la domótica de las viviendas, así como resolver dudas y aportar información [2].

La capacidad del asistente virtual con voz para resolver dudas y aportar información en el aula, como apoyo del profesorado al impartir la clase, es el objeto de esta investigación. Puesto que el número de alumnos dentro del aula es muy elevado, el uso de dichos asistentes facilita la labor del profesor, ya que no siempre puede estar disponible para resolver las dudas de todos. Otra ventaja que tiene el uso de estos asistentes es que cualquier pregunta planteada por el alumnado, por ingeniosa que sea, siempre va a tener una respuesta. Además, el hecho de que el alumnado tenga la tranquilidad de no tener que preguntarle directamente al profesor por miedo al ridículo y poder resolver la duda con el asistente virtual, hace que se incentive la participación de todos, además de resolver un gran número de dudas y favorecer el aprendizaje. El fin último es por tanto que el profesorado pueda contar con la ayuda de un asistente virtual con voz que se ponga a disposición del alumnado durante las sesiones didácticas. 


\section{Materiales}

Para poder llevar a cabo el estudio se analizan los diferentes asistentes virtuales disponibles en el mercado. Los ejemplos más destacados son Alexa de Amazon, Google Assistant, Cortana de Microsoft, DuerOS de Baidu, Siri de Apple, Bixby de Samsung y Watson [2]. De entre todos ellos, debido a su gran popularidad y gran adquisición por parte de los consumidores, en este trabajo se decide usar Alexa y Google Assistant.

El asistente virtual Alexa está disponible en todos los altavoces inteligentes de Amazon. El primero de estos altavoces se dio a conocer con el nombre de Amazon Echo o directamente Echo. Este altavoz estaba integrado por el asistente virtual Alexa. Dicho dispositivo salió por primera vez a la venta en noviembre de 2014, pero su comercialización se limitó a ciertos usuarios con cuentas Amazon Prime. Algo más tarde, en el año 2015 salió a la venta en Estados Unidos para el público en general [3]. Un año después surgió el modelo Echo Dot, siendo la versión de Echo con el tamaño más pequeño. El Echo Dot ( $3^{\mathrm{a}}$ generación), el cual se usa en este estudio, se puso a la venta en España el 16 de octubre de 2019. Este dispositivo está a la venta con y sin reloj. El coste que tiene la versión sin reloj es de 59,99 € y de 69,99 € la versión con reloj.

Para poner en funcionamiento este dispositivo se necesita una toma de corriente y una red de datos WiFi. Si es la primera vez que se va a poner en marcha dicho dispositivo, los pasos a seguir son los siguientes. Primero hay que descargar la última versión de la app Amazon Alexa. Después, es necesario conectar el Echo Dot usando el adaptador de corriente a un enchufe. El círculo de luz situado alrededor de la parte superior se iluminará de color azul, y al cabo de un minuto Alexa saludará y dará las siguientes pautas para llevar a cabo la instalación del asistente desde la app. Una vez configurado el dispositivo, para empezar a usarlo tan solo hay que decir la palabra "Alexa", y a partir de aquí el aparato se activa poniéndose en modo escucha para recibir la siguiente orden. Una vez recibida la orden, el dispositivo reconoce la pregunta y facilita una respuesta adecuada a la pregunta. En el caso de no tener una respuesta para la pregunta propuesta, el dispositivo lo indicará.

Hay dos elementos fundamentales de los que dependen las funciones de Alexa:

- Comandos de voz: estos comandos están integrados por defecto en el dispositivo y gracias a ellos se les puede hacer diversas peticiones.

- Skills: son unos complementos adicionales que pueden instalarse para incorporar más funcionalidades al dispositivo.

A través de los comandos de voz se le pueden hacer a Alexa una gran variedad de peticiones como por ejemplo solicitarle datos meteorológicos, pedirle información sobre diferentes temas o realizar una búsqueda sobre algunas cuestiones sobre un determinado producto o persona. El dispositivo, a través de los comandos, también ofrece la opción de pedirle la configuración de alarmas, iniciar cronómetros, programar recordatorios y preguntarle qué día es. Otra opción que incorpora Alexa es la posibilidad de hacer compras a través de la voz en Amazon y saber en qué estado se encuentra el pedido realizado. 
Por otro lado, las skills son aplicaciones específicamente creadas para Alexa pero diseñadas por terceros. Con las skills las funcionalidades del dispositivo aumentan, ya que con ellas se añaden más comandos de voz además de otras fuentes de consulta. A nivel mundial hay disponibles más de 25.000 skills, aunque de estas una minoría tienen como idioma el español.

El asistente virtual Google Assistant está disponible en los altavoces inteligentes de Google. El primero modelo de altavoz de esta marca fue el Google Home y se puso a la venta en 2016. La versión Google Home Mini salió a la venta en el año 2017 para competir con el Echo Dot ya que sus características, tamaño y precio eran muy similares. En este estudio se hace uso del altavoz Google Nest Mini, puesto a la venta en España el 22 de octubre de 2019 y cuyo precio es $59 €$.

Para poner en funcionamiento este dispositivo se necesita una toma de corriente y una red de datos WiFi. Si es la primera vez que se va a poner en marcha dicho dispositivo, los pasos a seguir son los siguientes. Primero se debe conectar el adaptador de corriente al Google Nest Mini. Después, hay que descargar la aplicación Google Home y a continuación seguir los pasos que aparecen en la pantalla del dispositivo para terminar la configuración. Una vez configurado el dispositivo, para empezar a usarlo tan solo hay que decir la palabra "Ok Google" y a partir de aquí el aparato se activa poniéndose en modo escucha para recibir la siguiente orden. Una vez recibida la orden, el dispositivo reconoce la petición y facilita una respuesta para la misma. En el caso de no tener una respuesta para la pregunta realizada el dispositivo se lo indicará al usuario.

Google Assistant permite que se pueda tener con dicho asistente una conversación usando un lenguaje natural. Se le puede pedir chistes, que lea poemas, cante canciones, etc., con el fin de entretener al usuario. Con este asistente virtual también es posible controlar mediante la voz dispositivos domóticos del hogar como pueden ser luces, persianas, termostatos, etc. Algunas de las funciones básicas que puede realizar este asistente son la búsqueda de información, la localización de alojamientos o restaurantes, informar de resultados deportivos, gestionar el calendario o realizar operaciones matemáticas entre otras opciones [4].

\section{Métodos}

En el presente estudio es necesario llevar a cabo un proceso de implantación de los asistentes virtuales con voz en el aula, lo que nos permitirá valorar la eficacia en la implantación de los mismos en el ámbito educativo. Se decide, de conformidad con el profesorado participante en el estudio, la utilización de los asistentes virtuales Echo Dot y Google Nest Mini. Para conocer el grado de satisfacción y validez en el uso de estos asistentes se propone una actividad didáctica que involucra el uso de los mismos. Dicha actividad consiste en el planteamiento de una batería de preguntas a los asistentes virtuales por parte de los estudiantes participantes.

Un total de 58 alumnos pertenecientes al nivel educativo de $2^{\circ}$ E.S.O., con edades comprendidas entre trece y catorce años, participan en el estudio. A través de la 
plataforma web docente, el alumnado participa en una actividad donde se les propone que planteen cualquier pregunta, duda o curiosidad que tengan de la materia impartida en la asignatura de Tecnología durante el curso académico 2019-20. Para desarrollarla, se les da un plazo de 3 días y esta actividad puntuará de forma equitativa dentro del $20 \%$ de la nota final otorgada para actividades. La participación de los alumnos en la actividad es muy satisfactoria, obteniendo un total de 58 consultas, una por alumno, que plantear a cada uno de los asistentes virtuales. Una vez obtenidas todas las preguntas o dudas de todo el alumnado, se procede a agrupar dichas preguntas en conceptuales, procedimentales y actitudinales para posteriormente formulárselas a cada uno de los asistentes virtuales escogidos para este estudio, es decir, el Google Nest Mini y el Echo Dot.

Una vez transcritas las respuestas de cada asistente son transmitidas a cada estudiante a través de la plataforma docente online. Posteriormente, a través de la misma plataforma se le entrega a todo el alumnado un cuestionario para comprobar si la consulta realizada en un principio ha sido resuelta. En caso de haber sido resuelta, se comprueba si la respuesta ha aclarado su pregunta o duda, y en caso de haber sido resuelta por los dos asistentes, comprobar qué respuesta les ha sido más útil. Asimismo, todas las respuestas obtenidas por los asistentes son contrastadas por los autores, para valorar si es adecuado o no su uso para la asignatura de Tecnología, además de comprobar para qué tipo de preguntas es capaz de dar más repuestas cada uno de los asistentes, comprobando la exactitud y eficacia de la misma.

\section{Resultados y discusión}

El presente estudio trata de medir la eficacia de los asistentes virtuales con voz en el aula como apoyo al docente en el transcurso de la enseñanza. Sin embargo, la falta de variedad de estudios preliminares en este ámbito dificulta la comparativa con enfoques previos. El uso de asistentes virtuales dentro del aula se presenta como una posible opción de futuro para implementarse dentro del ámbito educativo y así facilitar y asistir en la labor docente del profesor.

Las dudas planteadas por el alumnado se han agrupado según el tipo de contenido al que refieren: conceptual, procedimental y actitudinal. Por una parte, el análisis de los resultados muestra que de las 58 preguntas planteadas por los estudiantes ningún asistente fue capaz de responder más del $56,9 \%$ de las mismas, incluyendo las tres tipologías. Este resultado puede deberse a la falta de contexto general y la falta de entrenamiento en el contexto educativo de dichos asistentes. Otro dato destacable es la cantidad de respuestas válidas que arrojan los asistentes, puesto que el hecho de poder dar una respuesta no es sinónimo de que ésta sea válida y, por tanto, sirva de aclaración de las posibles dudas planteadas por los alumnos. Considerando este aspecto, solo 20 (Google) y 14 (Amazon) respuestas se demuestran como válidas del total de 58 preguntas planteadas por el alumnado. De entre las diferentes categorías de preguntas, se ha observado que las respuestas para preguntas de tipo procedimental y actitudinal son en las que más error generan con independencia del dispositivo. La validez de las respuestas, está basada en el criterio de los autores de este estudio. 
Se observa que los asistentes presentan serias limitaciones cuando la pregunta planteada no es clara, directa o concisa. Asimismo, dichas limitaciones aparecen cuando toman parte de la información de la pregunta, esto es, palabras clave, que no necesariamente responden a la cuestión planteada. En muchos de estos casos, la respuesta ofrece información relacionada pero que poco o nada tiene que ver con la duda. Este hecho hace que, en general, sean más eficaces respondiendo dudas de carácter conceptual (principalmente para teoría) donde las posibles interpretaciones son escasas.

Considerando que la materia de tecnología tiene, desde un punto de vista docente, un carácter principalmente práctico, nos presenta la seria duda de si el uso de estos asistentes a día de hoy es recomendable. No obstante, sí que se ha observado un efecto positivo de cara a motivar al alumnado. El 100\% de los alumnos ha participado en la actividad propuesta, no existiendo una preferencia clara por uno u otro dispositivo. Sí que hubo una respuesta clara ante la pregunta de si querrían usar los dispositivos durante el próximo curso llegando a contestar afirmativamente un 94,8\% del alumnado.

\section{Conclusiones}

La aparición de los asistentes virtuales puede jugar un factor importante en cómo asimilamos la información a la que dichos dispositivos nos ponen al alcance. La barrera técnica cada vez es menor y la conversación con el aparato puede hacer más asequible y romper ciertas barreras de comportamiento social como la timidez y la inseguridad. En el campo docente estos dispositivos pueden ser de gran apoyo y, en ese contexto, se ha desarrollado una experiencia práctica para comprobar su efectividad. Si bien es cierto que todavía cierto tipo de consultas no son respondidas de manera satisfactoria, la acogida por el alumnado ha sido muy positiva. Es responsabilidad de los docentes ser conscientes de su presencia e integrarlos en la práctica docente para guiar hacia un uso correcto y adecuado de este tipo de asistentes.

\section{Referencias}

1. Huertas, A. y Pantoja, A. (2016). Efectos de un programa educativo basado en el uso de las TIC sobre el rendimiento académico y la motivación del alumnado en la asignatura de tecnología de Educación Secundaria. Recuperado de https://www.redalyc.org/articulo.oa?id=70645811009

2. Liege, J. y Lostalé, E. (2018). La era de la voz: Asistentes Virtuales y Voice Marketing. Recuperado de https://www.kanlli.com/wpcontent/uploads/2018/09/LA_ERA_DE_LA_VOZ_ASISTENTES_VIRTUALES_Y VOIC E_MARKETING.pdf

3. Scholz, M. M. (2019). Google Actions en entornos eHealth. Recuperado de https://riull.ull.es/xmlui/bitstream/handle/915/14534/Google\%20Actions\%20en\%20entornos $\% 20$ ehealth.pdf?sequence $=1$

4. CTI. (2019). Todo el mundo habla de los asistente virtuales, pero ¿cómo los utilizan realmente los usuarios? Recuperado de https://aura.telefonica.com/public/papers/04-todo-el-mundohabla-sobre-asistentes-virtuales-es.pdf 